In European Trans. Telecommunications, Special Issue on CDMA

Techniques for Wireless Communication Systems, vol. 9, no. 5, pp. 403-418, Sept./Oct. 1998. (invited paper)

\title{
Joint Intersymbol and Multiple-Access Interference Suppression Algorithms for CDMA Systems
}

\author{
Soosan Beheshti, Steven H. Isabelle, and Gregory W. Wornell,
}

February 1998

\begin{abstract}
Two promising classes of techniques are developed for efficient multiuser detection in codedivision multiple-access (CDMA) communication systems subject to fading due to time-varying multipath propagation. Both are designed to jointly suppress both intersymbol and multipleaccess interference inherent in such systems, and exploit all available time and frequency diversity.

The first is a family of linear receivers for time-varying multiuser channels that generalize familiar linear equalizers designed for traditional single-user linear time-invariant channels. Minimum mean-square error, zero-forcing, and matched-filter versions of such multiuser detectors are all developed within a common state-space framework, and have convenient recursive implementations. Performance issues as well as natural decision-feedback variants of the detector structure are both discussed.

The second is a family of nonlinear receivers that are specifically designed for use with spread-signature CDMA systems on time-varying multipath channels. These multiuser detectors employ a batch-iterative (multipass) decoding algorithm based on a successive-cancellation strategy. Several aspects of the performance of this algorithm are developed, including its monotonic convergence property.

Collectively, these two classes of algorithmic structures for joint equalization, interference suppression, demodulation, and detection are representative of several emerging and interrelated approaches to receiver design for next-generation CDMA systems.

Index Terms - code-division multiple-access, wireless communications, fading channels, equalization, spread-response precoding, spread-signature CDMA, multiuser detection, interference cancellation, iterative decoding, stripping, multipass algorithms
\end{abstract}

\section{Introduction}

In a wide-range of wireless communication applications, there is a need for users to be able to communicate efficiently and asynchronously among themselves in the presence of fading due to

This work has been supported in part by the Office of Naval Research under Grant No. N00014-96-1-0930, the National Science Foundation under Grant No. MIP-9502885, and the Army Research Laboratory under Cooperative Agreement DAAL01-96-2-0002.

S. Beheshti and G. W. Wornell are with the Department of Electrical Engineering and Computer Science, Massachusetts Institute of Technology, Cambridge, MA 02139, as was S. H. Isabelle. S. H. Isabelle is now with Solana Technology Corp., San Diego, CA 92122. 
multipath propagation. For example, in a cellular mobile radio environment, the transmissions of the individual mobiles pass through generally distinct channels, and a noisy version of their superposition is obtained at the base station. In such scenarios, obtaining reliable estimates of the symbols transmitted by a particular user (or all users) requires the mitigation of several sources of interference.

In CDMA systems, where all users spread their transmission over a common transmission bandwidth, a dominant impairment is interference between users, referred to as multiple-access interference. There is a substantial literature on the problem of multiple-access interference suppression in wireless systems, and a wide range of efficient algorithms have been proposed for use in receivers - see, e.g., [1] [2] [3] [4], as well as [5] and the references therein. In much of this literature, attention is restricted to the case where there is no intersymbol interference. ${ }^{1}$ Unfortunately, this strongly limits the utility of such algorithms. Indeed, to obtain one of the most important advantages of CDMA systems over traditional systems, these systems need to be used with bandwidths large enough to ensure that intersymbol interference is present.

The reason is that reliable communication in the presence of fading requires that diversity of some form be exploited to improve both average and worst-case performance (e.g., mean bit-error rate and outage probability, respectively) [6]. Intersymbol interference is an important source of such diversity in wireless systems - in particular, frequency diversity. In CDMA systems, for example, such interference arises when the total transmission bandwidth is large compared to the coherence bandwidth of the channel, so that not all frequencies within the transmission fade in unison. From this perspective, equalization or intersymbol interference suppression more generally are the means by which frequency diversity is exploited to improve reliability.

In a similar manner, more recently introduced spread-signature CDMA systems [7] [8] and spread-response precoding systems [9] are designed to enable a time diversity benefit to be obtained, either alone or in conjunction with a frequency diversity benefit. With these systems, in addition to any spectral spreading, users temporally spread the transmission of their symbols beyond the coherence time of the channel by deliberately introducing intersymbol interference. In this case, too, suitably designed intersymbol interference suppression allows such diversity to be exploited.

Finally, in antenna precoding systems such as those developed in [10], spatial diversity is obtained through the use of a multiple-element transmitter antenna array (and can be used in conjunction with other forms of diversity). Moreover, when used in combination with suitably designed signal processing [10], these systems also have the effect of transforming this diversity into intersymbol interference. Again, intersymbol interference suppression is required to exploit this diversity.

In this paper we develop novel linear and nonlinear algorithms suitable for use in the receivers of these classes of systems. These algorithms for equalization, demodulation, and decoding jointly suppress both multiple-access and intersymbol interference and therefore exploit the inherent diversity. Moreover, as will become apparent, in environments without intersymbol interference the resulting algorithms specialize to methods closely related to some classical approaches to multiuser detection.

An outline of the paper is as follows. In Section 2, we outline a suitable mathematical model for the system of interest. In Section 3, we develop linear multiuser equalization strategies that

\footnotetext{
${ }^{1}$ In this work we use the terms "intersymbol interference" and "interchip interference" interchangeably, although we point out that there are scenarios outside the scope of this paper where it is necessary to distinguish between the two.
} 
generalize both classical single-user linear and decision-feedback equalizers, and classical multiuser detectors. In Section 4 we develop multipass receivers that implement iterative decoding, suppressing interference by a successive-cancellation (stripping) strategy. Finally, Section 5 contains some concluding remarks and outlines some potentially fruitful directions for further research.

\section{System Model}

We consider a passband CDMA system in which there are $M$ users, each transmitting a white data stream at rate $\mathcal{W}_{0}$, and all sharing a total fixed bandwidth $L \mathcal{W}_{0}$ with $L \geq M$. Thus, the bandwidth expansion factor $L / M$ is a measure of the excess bandwidth per user. In the equivalent discrete-time baseband model for the system, the modulation process can be viewed as follows. The coded symbol stream of the $m$ th user $(1 \leq m \leq M)$, which we denote by $x_{m}[n]$, has power $\mathcal{E}_{m}$ and is modulated onto a unique signature sequence $h_{m}[n]$ to produce $y_{m}[n]$ which is transmitted within the total available bandwidth. For simplicity of exposition, we assume throughout that the same signature is used for each symbol of a particular user's transmission.

Conceptually, it is convenient to view the modulation process in two stages. As depicted in Fig. 1, these stages correspond to upsampling (i.e., zero-insertion) by a factor $L$, followed by linear time-invariant filtering with the signature sequence, i.e.,

$$
y_{m}[n]=\sum_{k} x_{m}[k] h_{m}[n-k L]
$$

We emphasize at the outset that we avoid imposing any constraint on the length $K$ of the signature sequences in this system. In so doing, our results apply not only to conventional CDMA systems, for which $K=L$, but also to the spread-signature CDMA systems mentioned in the introduction, for which $K>L$.

Often - but certainly not always - the signatures are chosen to satisfy some convenient orthogonality properties. For example, often the $h_{m}[n]$ are chosen so that they form an orthonormal set, i.e.,

$$
\sum_{k} h_{i}[k-n L] h_{l}[k-m L]=\delta[n-m] \delta[i-l]
$$

where $\delta[\cdot]$ is the unit-sample, i.e.,

$$
\delta[k]=\left\{\begin{array}{ll}
1 & k=0 \\
0 & \text { otherwise }
\end{array},\right.
$$

in which case all the symbols of all the users are modulated on orthogonal, unit-energy waveforms.

Note that most familiar multiple-access techniques fit into this framework-i.e., in addition to CDMA, both time-division multiple-access (TDMA) and frequency-division multiple-access (FDMA) systems have implementations of the form depicted in Fig. 1 and have signatures satisfying (2). Moreover, spread-signature CDMA systems having binary-valued coefficients $\left(h_{m}[n]= \pm K^{-1 / 2}\right)$ that satisfy (2) also exist; these are the maximally-spread signature sets developed in [7].

We emphasize, however, that we will not assume that (2) is satisfied in our initial development. In fact, in many cases the users can share even the same signature without impacting performance; 
for example, users may be distinguished by the channels their respective transmissions pass through. In any event, we assume that the signature sequences are all known at the receiver.

The multiuser channel we consider, which is depicted in Fig. 2, corresponds to a rather general intersymbol interference environment. In this model, the complex-valued and, in general, time-varying channel impulse response experienced by the $m$ th user's transmission is $a_{m}[n ; k]$, the response at time $n$ to a unit sample input at time $k$. Hence, the sequence obtained at the receiver is

$$
r[n]=\sum_{m=1}^{M} \sum_{k} a_{m}[n ; k] y_{m}[n-k]+w[n],
$$

where $w[n]$ is a zero-mean, complex-valued, stationary, circularly symmetric, white Gaussian noise sequence with variance

$$
E\left[|w[n]|^{2}\right]=\mathcal{N}_{0} \mathcal{W}_{0}
$$

The channel kernels $a_{m}[n ; k]$ take into account both the physical propagation medium and path losses, as well as the relative delays among the users transmissions due to the inherent asynchrony in the system. We will frequency restrict our attention to the case in which the $a_{m}[n ; k]$ are causal, so that $a_{m}[n ; k]=0$ for $k<0$, and finite length, i.e., $a_{m}[n ; k]=0$ for $k \geq J$.

When the channel is time-selective but frequency-nonselective (and delays are multiples of the chip time), the $a_{m}[n ; k]$ take the form

$$
a_{m}[n ; k]=a_{m}[n] \delta\left[k-n_{m}\right],
$$

where $n_{m}$ is the associated delay. In this case, the received signal (3) specializes to

$$
r[n]=\sum_{m=1}^{M} a_{m}[n] y_{m}\left[n-n_{m}\right]+w[n],
$$

and only time diversity (in the form of, for example, spread-signature CDMA) can be exploited to combat fading.

On the other hand, when the channel is time-nonselective but frequency-selective, the $a_{m}[n ; k]$ are independent of $n$, corresponding to a time-invariant, intersymbol interference channel with constituent unit-sample responses

$$
a_{m}[k] \triangleq a_{m}[0 ; k]=a_{m}[n ; k]
$$

In this case, the received signal (3) takes the form

$$
r[n]=\sum_{m=1}^{M} \sum_{k} a_{m}[k] y_{m}[n-k]+w[n],
$$

and only frequency diversity can be exploited to combat fading.

In the sequel, it will be convenient to combine the signature modulation process with the effects of the channel to obtain an equivalent model in which the symbol streams of the individual users 
can be viewed as time-division multiplexed before being transmitted over a multiuser channel where the unit-sample responses are now

$$
\tilde{a}_{m}[n ; k]=\sum_{l} a_{m}[n ; l] h_{m}[k-l],
$$

which in the time-invariant case specializes to

$$
\tilde{a}_{m}[n]=a_{m}[n] * h_{m}[n] .
$$

The channel model described in this section is a reasonable one for both forward-link and reverse-link transmission within a single cell of a typical cellular multiple-access system in which there are $M$ mobiles and a single base station. For forward-link (base-to-mobile) transmission, the $M$ transmissions are multiplexed at the base station before being broadcast over the channel, so what is received at a particular mobile is the set of coordinated (synchronous) transmissions distorted by a common channel, i.e.,

$$
a_{1}[n ; k]=a_{2}[n ; k]=\cdots=a_{M}[n ; k] \triangleq a[n ; k],
$$

from which it must extract its own message. By contrast, in the case of reverse-link (mobile-tobase) transmission, the $M$ transmissions from the individual mobiles experience generally different channels before being received superimposed at the base station. Moreover, provided the mobiles are reasonably well-separated, the associated channels $a_{1}[n ; k], a_{2}[n ; k], \ldots, a_{M}[n ; k]$ can be modeled as mutually independent.

In both scenarios, the decoding problem we consider is then one of making decisions $\breve{x}_{m}[n]$ of the symbol streams of the constituent users from a received signal $r[n]$ of the general form (3). We restrict our attention to the case in which the $a_{m}[n ; k]$ are all known at the receiver; in practice, estimates of these coefficients can be obtained either through the use of a training-data or pilot-tone based approach, or using a blind algorithm.

\section{Linear Multiuser Equalization}

We now turn our attention to developing equalizers for the multiuser channel that generalize the familiar linear equalizers designed for traditional single-user linear time-invariant channels. To facilitate the development of these equalizers, it is convenient to express the received signal as an observation of the state of a multiple-input-multiple-output linear system. Because of the upsampling inherent in the modulation process, polyphase decompositions play a key role in the state-space description, as we now develop.

\subsection{Polyphase Decompositions and State-Space Models}

The $L$ th order polyphase decomposition of an arbitrary signal $p[n]$ is the vector of sequences

$$
\mathbf{p}[n] \triangleq[p[n L] \quad p[n L+1] \quad \cdots \quad p[n L+L-1]]^{\mathrm{T}} .
$$


The polyphase decomposition of a general time-varying channel response $q[n ; k]$ is the vector of kernels

$$
\mathbf{q}[n ; k] \triangleq[q[n L ; k L] \quad q[n L+1 ; k L+1] \quad \cdots \quad q[n L+L-1 ; k L+L-1]]^{\mathrm{T}} .
$$

Note that (12) implies that the polyphase decomposition of a specifically time-invariant kernel $q[n]$ has the form

$$
\mathbf{q}[n]=\left[\begin{array}{llll}
q[n L] & q[n L+1] & \cdots & q[n L+L-1]
\end{array}\right]^{\mathrm{T}} .
$$

With this notation the polyphase decomposition $\mathbf{r}[n]$ of the received signal $r[n]$ can be expressed in terms of the input symbols $x_{m}[n]$ and the polyphase components $\tilde{\mathbf{a}}_{m}[n ; k]$ of the composite channels $\tilde{a}_{m}[n ; k]$ via

$$
\mathbf{r}[n]=\sum_{i=1}^{M} \sum_{k} \tilde{\mathbf{a}}_{i}[n ; k] x_{i}[n-k]+\mathbf{w}[n]=\sum_{k} \tilde{\mathbf{a}}[n ; k] \mathbf{x}[n-k]+\mathbf{w}[n],
$$

where

$$
\mathbf{x}[n]=\left[\begin{array}{llll}
x_{1}[n] & x_{2}[n] & \cdots & \left.x_{M}[n]\right]^{\mathrm{T}}
\end{array}\right.
$$

is the collection of $M$ symbols transmitted by the collection of users at time $n, \mathbf{w}[n]$ is the polyphase representation for the receiver noise $w[n]$, and

$$
\tilde{\mathbf{a}}[n ; k]=\left[\begin{array}{llll}
\tilde{\mathbf{a}}_{1}[n ; k] & \tilde{\mathbf{a}}_{2}[n ; k] & \cdots & \left.\tilde{\mathbf{a}}_{M}[n ; k]\right]
\end{array} .\right.
$$

With this notation, we can write the polyphase decomposition of the received signal as the output of a linear dynamical system of the form

$$
\begin{aligned}
\mathbf{s}[n+1] & =\mathbf{F} \mathbf{s}[n]+\mathbf{G} \mathbf{x}[n+1] \\
\mathbf{r}[n] & =\mathbf{A}[n] \mathbf{s}[n]+\mathbf{w}[n],
\end{aligned}
$$

where the state $\mathbf{s}[n]$ is a $\tilde{K} M$-dimensional vector of the form

$$
\mathbf{s}[n]=\left[\begin{array}{c}
\mathbf{x}[n] \\
\mathbf{x}[n-1] \\
\vdots \\
\mathbf{x}[n-\tilde{K}+1]
\end{array}\right]
$$

with

$$
\tilde{K}=\left\lceil\frac{J+K-1}{L}\right\rceil
$$

denoting the effective length of the polyphase components of the time-varying channel responses 
$\tilde{a}_{m}[n ; k]$. In the state equation (15a), we therefore have, with $\mathbf{I}$ denoting the identity matrix,

$$
\mathbf{F}=\left[\begin{array}{ccccc}
\mathbf{0} & \mathbf{0} & \mathbf{0} & \cdots & \mathbf{0} \\
\mathbf{I} & \mathbf{0} & \mathbf{0} & \cdots & \mathbf{0} \\
\mathbf{0} & \mathbf{I} & \mathbf{0} & \cdots & \mathbf{0} \\
\vdots & \vdots & \vdots & \ddots & \vdots \\
\mathbf{0} & \mathbf{0} & \cdots & \mathbf{I} & \mathbf{0}
\end{array}\right], \quad \mathbf{G}=\left[\begin{array}{c}
\mathbf{I} \\
\mathbf{0} \\
\mathbf{0} \\
\vdots \\
\mathbf{0}
\end{array}\right]
$$

where the blocks in $\mathbf{F}$ and $\mathbf{G}$ are of size $M \times M$, while in the observation equation (15b) we have that $\mathbf{A}[n]$ is the following $L \times \tilde{K} M$ matrix containing the channel and signature information:

$$
\mathbf{A}[n]=[\tilde{\mathbf{a}}[n ; 0] \quad \tilde{\mathbf{a}}[n ; 1] \quad \cdots \quad \tilde{\mathbf{a}}[n ; \tilde{K}-1]] .
$$

With the users uncorrelated and transmitting white symbol streams, we have

$$
E\left[\mathbf{x}[n] \mathbf{x}^{\dagger}[m]\right]=\mathbf{E} \delta[n-m]
$$

where

$$
\mathbf{E}=\operatorname{diag}(\mathbf{D}, \mathbf{D}, \ldots, \mathbf{D})
$$

with

$$
\mathbf{D}=\operatorname{diag}\left(\mathcal{E}_{1}, \mathcal{E}_{2}, \ldots, \mathcal{E}_{M}\right)
$$

Note that in the time-invariant channel case, the observation matrix $\mathbf{A}[n]$ is a constant $\mathbf{A}$, i.e., independent of $n$. We also note that a state-space model of the form (15) also applies when in addition there is a multiple-element antenna array at the receiver in the system. Although not explicitly developed in this paper, these extensions are obtained via a straightforward augmentation of the observation vectors.

\subsection{Recursive MMSE Equalizers}

A linear MMSE equalizer for the multiple-access system described by (15) requires the constuction of linear MMSE estimates of the state (16). Such estimates can be computed sequentially and efficiently via the Kalman filtering algorithm. Denoting by $\hat{\mathbf{s}}[n \mid k]$ the estimate of the state at time $n$ given observations of $\mathbf{r}[l]$ up to time $k$-and denoting the associated error covariance by $\boldsymbol{\Lambda}[n \mid k]$, the state estimation (equalizer) equations take the form [11] [12]

$$
\begin{aligned}
\hat{\mathbf{s}}[n \mid n] & =\mathbf{F} \hat{\mathbf{s}}[n-1 \mid n-1]+\boldsymbol{\mu}[n](\mathbf{r}[n]-\mathbf{A}[n] \mathbf{F} \hat{\mathbf{s}}[n-1 \mid n-1]) \\
\boldsymbol{\mu}[n] & =\boldsymbol{\Lambda}[n \mid n-1] \mathbf{A}^{\dagger}[n]\left(\mathbf{A}[n] \boldsymbol{\Lambda}[n \mid n-1] \mathbf{A}^{\dagger}[n]+\mathcal{N}_{0} \mathcal{W}_{0} \mathbf{I}\right)^{-1} \\
\boldsymbol{\Lambda}[n \mid n] & =(\mathbf{I}-\boldsymbol{\mu}[n] \mathbf{A}[n]) \boldsymbol{\Lambda}[n \mid n-1] \\
\boldsymbol{\Lambda}[n+1 \mid n] & =\mathbf{F} \boldsymbol{\Lambda}[n \mid n] \mathbf{F}^{\mathrm{T}}+\mathbf{G} \mathbf{E} \mathbf{G}^{\mathrm{T}},
\end{aligned}
$$


and are initialized with $\hat{\mathbf{s}}[-1 \mid-1]=\mathbf{0}$ and $\boldsymbol{\Lambda}[-1 \mid-1]=\mathbf{0}$. Note that this initialization is appropriate when transmission commences at $n=0$; for other possible initializations, see [13]. Note too that although we do not need to compute it explicitly, the one-step prediction is given by

$$
\hat{\mathbf{s}}[n+1 \mid n]=\mathbf{F} \hat{\mathbf{s}}[n \mid n] .
$$

There are several notable features of these estimation equations. First, because of the form of the state vector (16) smoothed estimates of the symbols $\mathbf{x}[n-k]$ for $1 \leq k \leq \tilde{K}-1$ are available at the same time as a filtered estimate of $\mathbf{x}[n]$ is computed. In particular, these estimates of the symbol streams of the users are given by

$$
\hat{\mathbf{x}}[n-k \mid n]=\boldsymbol{\Delta}[k] \hat{\mathbf{s}}[n \mid n], \quad 0 \leq k \leq \tilde{K}-1
$$

where

$$
\boldsymbol{\Delta}[k]=\left[\begin{array}{llll}
\mathbf{I} \delta[k] & \mathbf{I} \delta[k-1] & \cdots & \mathbf{I} \delta[k-\tilde{K}+1] .]
\end{array}\right.
$$

These smoothed estimates are available, in effect, for free - no additional computation is required.

We also remark that further smoothed estimates can be obtained by augmenting the state vector (16) with additional lags of the transmitted symbols, i.e.,

$$
\mathbf{s}^{\prime}[n]=\left[\begin{array}{c}
\mathbf{s}[n] \\
\mathbf{x}[n-\tilde{K}] \\
\mathbf{x}[n-\tilde{K}-1] \\
\vdots \\
\mathbf{x}\left[n-\tilde{K}^{\prime}+1\right]
\end{array}\right]
$$

and modifying the state equations (15) by augmenting the matrices $\mathbf{F}$ and $\mathbf{A}[n]$ to obtain $\mathbf{F}^{\prime}$ and $\mathbf{A}^{\prime}[n]$, respectively. In practice, computationally more efficient algorithms can generally be used to obtain these additional smoothed estimates.

Finally, note that the algorithm requires that the covariance of the estimation error $\boldsymbol{\Lambda}[k \mid k]$ be computed at each time step. This reliability information can potentially be used to enhance the performance of a higher level error correction scheme (involving, for example, soft-decision decoding).

Other related equalizer structures can also be related to (20). For example, a recursive implementation of the zero-forcing multiuser equalizer is obtained by replacing the gain (20b) with

$$
\boldsymbol{\mu}[n]=\boldsymbol{\Lambda}[n \mid n-1] \mathbf{A}^{\dagger}[n]\left(\mathbf{A}[n] \mathbf{\Lambda}[n \mid n-1] \mathbf{A}^{\dagger}[n]\right)^{-1} .
$$

A natural generalization of the single-user zero-forcing equalizer and decorrelating multiuser detector, this equalizer completely cancels both intersymbol and multiple-access interference, but at the expense of noise enhancement.

Similarly, a recursive implementation of the matched-filter multiuser equalizer is obtained by 
using the gain

$$
\boldsymbol{\mu}[n]=\frac{\boldsymbol{\Lambda}[n \mid n-1] \mathbf{A}^{\dagger}[n]}{\mathcal{N}_{0} \mathcal{W}_{0}}
$$

This receiver ignores intersymbol and multiple-access interference and focuses only on noise suppression.

It is important to emphasize that in both these cases the matrices $\boldsymbol{\Lambda}[n \mid n]$ and $\boldsymbol{\Lambda}[n+1 \mid n]$ no longer correspond to the associated estimation errors, except for limiting cases: $\mathcal{N}_{0} \mathcal{W}_{0} \rightarrow 0$ in the zero-forcing case, and $\mathcal{N}_{0} \mathcal{W}_{0} \rightarrow \infty$ in the matched-filter case.

\subsection{Steady-State Equalization Error}

When the channel is time-invariant and the equalization algorithm is operating in the steady-state, the state update equations reduce to

$$
\begin{aligned}
\hat{\mathbf{s}}[n \mid n] & =\mathbf{F} \hat{\mathbf{s}}[n-1 \mid n-1]+\boldsymbol{\mu}(\mathbf{r}[n]-\mathbf{A} \mathbf{F} \hat{\mathbf{s}}[n-1 \mid n-1]) \\
\boldsymbol{\mu} & =\boldsymbol{\Sigma}_{\infty} \mathbf{A}^{\dagger}\left(\mathbf{A} \boldsymbol{\Sigma}_{\infty} \mathbf{A}^{\dagger}+\mathcal{N}_{0} \mathcal{W}_{0} \mathbf{I}\right)^{-1}
\end{aligned}
$$

where

$$
\boldsymbol{\Sigma}_{\infty}=\lim _{n \rightarrow \infty} \boldsymbol{\Lambda}[n+1 \mid n]
$$

is steady-state prediction error covariance. This covariance is obtained as the solution to the Ricatti equation

$$
\boldsymbol{\Sigma}_{\infty}=\mathbf{F}\left(\boldsymbol{\Sigma}_{\infty}^{-1}+\left(\mathcal{N}_{0} \mathcal{W}_{0}\right)^{-1} \mathbf{A}^{\dagger} \mathbf{A}\right)^{-1} \mathbf{F}^{\mathrm{T}}+\mathbf{G} \mathbf{E} \mathbf{G}^{\mathrm{T}}
$$

which can be obtained using any of a number of algorithms — see, e.g., [11]. The associated steadystate filtering error covariance

$$
\boldsymbol{\Lambda}_{\infty}=\lim _{n \rightarrow \infty} \boldsymbol{\Lambda}[n \mid n]
$$

takes the form

$\boldsymbol{\Lambda}_{\infty}=(\mathbf{I}-\boldsymbol{\mu} \mathbf{A}) \boldsymbol{\Sigma}_{\infty}=\boldsymbol{\Sigma}_{\infty}-\boldsymbol{\Sigma}_{\infty} \mathbf{A}^{\dagger}\left(\mathbf{A} \boldsymbol{\Sigma}_{\infty} \mathbf{A}^{\dagger}+\mathcal{N}_{0} \mathcal{W}_{0} \mathbf{I}\right)^{-1} \mathbf{A} \boldsymbol{\Sigma}_{\infty}=\mathcal{N}_{0} \mathcal{W}_{0}\left(\mathcal{N}_{0} \mathcal{W}_{0} \boldsymbol{\Sigma}_{\infty}^{-1}+\mathbf{A}^{\dagger} \mathbf{A}\right)^{-1}$

where the second equality in (29) follows from (26b), and the third from an application of the matrix inversion lemma [12]. From (29) we see immediately that the MMSE multiuser equalizer is near-far resistant, i.e., $\boldsymbol{\Lambda}_{\infty} \rightarrow \mathbf{0}$ as $\mathcal{N}_{0} \mathcal{W}_{0} \rightarrow 0$. For the purposes of illustration, suppose that all users transmit at the same power, i.e., $\mathcal{E}_{1}=\mathcal{E}_{2}=\cdots=\mathcal{E}_{M} \triangleq \mathcal{E}$. Then (29) implies more generally that at high SNR the total normalized mean-square error falls off inversely with SNR:

$$
\operatorname{tr}\left(\frac{\boldsymbol{\Lambda}_{\infty}}{\mathcal{E}}\right) \approx\left[\frac{\mathcal{E} \operatorname{tr}\left(\mathbf{A}^{\dagger} \mathbf{A}\right)}{\mathcal{N}_{0} \mathcal{W}_{0}}\right]^{-1}
$$


Related expressions for the associated steady-state infinite-interval smoothing error covariance follow from the multichannel Wiener filtering results presented in [14].

\subsection{Example}

As an illustration of the performance of the algorithm, consider a simple example involving reverselink transmission from $M=2$ users using conventional CDMA signatures of length $K=L=4$. The signatures are orthogonal Hadamard sequences, and the two users transmit with equal power $\mathcal{E}$. The channels experienced by the two users are time-invariant and have real-valued unit-sample responses given by

\begin{tabular}{|c|rrrrr|}
\hline$n=$ & 0 & 1 & 2 & 3 & 4 \\
\hline$a_{0}[n]$ & 0.328 & 0.176 & 0.011 & 0.099 & -0.196 \\
$a_{1}[n]$ & 0.678 & 0.024 & 0.719 & 0.106 & 0.349 \\
\hline
\end{tabular}

The received powers are therefore quite asymmetric:

$$
\mathcal{E} \sum_{n}\left|a_{0}[n]\right|^{2}=0.187 \mathcal{E}, \quad \mathcal{E} \sum_{n}\left|a_{1}[n]\right|^{2}=1.110 \mathcal{E}
$$

The resulting equalization performance is depicted in Fig. 3. The top figures depict the frequency responses of the channels for each of the two users. The bottom left figure shows the combined mean-square equalization error in the signals for the two users after filtering. The nearfar resistance of the equalization is apparent - there is no interference-limited behavior at high SNR. The bottom right figure shows the additional reduction in mean-square equalization error that can be obtained through the use of smoothing. That there is comparatively little benefit to such smoothing beyond a couple lags is a consequence of the comparatively short channel responses involved.

We conclude this section with a couple of remarks. First, we emphasize that the equalization structures developed in this portion of the paper apply equally well to both conventional CDMA systems as well as more recently introduced spread-signature CDMA systems. The primary difference between the two in terms of equalizer implementation is that the latter class requires an appropriately larger state space. Second, as mentioned during the development, it is also important to recognize that the these same equalizer structures can be readily extended for use in narrowband or wideband systems employing multiple-element receiver antenna arrays. Finally, it is worth emphasizing that the algorithms developed above operate at the chip rate of the system. In practice, baud (symbol) rate algorithms are preferred as they generally have much lower computational requirements. Related baud-rate state-space equalizer structures are discussed in [13].

\subsection{Nonlinear Multiuser Equalization}

The simplest means for using the preceding linear multiuser equalizers to obtain (hard) symbol decisions is to follow the (soft) estimates $\hat{\mathbf{x}}[n-k \mid n]$ in (21) at the equalizer output with a simple memoryless decision device, i.e., a slicer. It is this slicer that exploits the property of digital communication systems that the transmitted symbols are drawn from a discrete alphabet. In practice, important performance improvements are obtained by exploiting this discrete-alphabet property in a more integrated manner in the receiver. 
A highly effective means for accomplishing this is to use maximum likelihood sequence detection to jointly decode all users, generalizing the corresponding approaches of [15] for single-user scenarios and [16] for multiuser ones. This generalization is discussed in more detail in, e.g., [13]. However, even when implemented using the associated Viterbi algorithms, the computational complexity inherent in such receivers is prohibitive in practice.

Another means for exploiting the discrete-alphabet property that requires substantially less computational complexity is to employ a decision-feedback structure, analogous to an approach used in single-user decision-feedback equalizers (DFE's).

Using our state-space framework, it is straightforward to develop a natural extension of the single-user MMSE DFE described in, e.g., [17]. We begin by noting that in the recursion (20a) for our linear MMSE equalizer, the state estimate is updated via two terms. The first is a prediction of the state based on the state estimate at the previous symbol time. Exploiting a DFE strategy, the soft state estimates in both terms of (20a) can be replaced with hard symbol decisions that are typically more accurate, especially at high SNR. With this approach, when, for example, the symbols are binary $x_{m}[n] \in\{-1,+1\}$, the decisions are generated recursively according to [cf. (20a)]

$$
\check{\mathbf{s}}[n \mid n]=\operatorname{sgn} \hat{\mathbf{s}}[n \mid n]=\operatorname{sgn}\{\mathbf{F} \check{\mathbf{s}}[n-1 \mid n-1]+\boldsymbol{\mu}[n](\mathbf{r}[n]-\mathbf{A}[n] \mathbf{F} \check{\mathbf{s}}[n-1 \mid n-1])\} .
$$

where

$$
\operatorname{sgn} v= \begin{cases}+1 & v \geq 0 \\ -1 & v<0\end{cases}
$$

is the associated slicer for this signal set. For other signal constellations, it suffices to substitute the appropriate slicer function in (32).

The DFE structure just described is one example of a sequential nonlinear multiuser equalization/decoding algorithm that is advantageous when the SNR is at least moderately large. However, a variety of other types of efficient nonlinear multiuser equalizer structures can also be developed.

We next explore one such alternative - an efficient batch-iterative equalization/decoding algorithm based on a successive-cancellation structure.

\section{Iterative Multiuser Decoding}

Instead of a decision-feedback algorithm in which symbols are decoded in a sequential manner, with past decisions incorporated to improve the accuracy of future decisions, another approach to decoding is to apply a multipass decoding algorithm. In the multipass strategy we develop in this part of the paper, a linear equalizer is applied to the full data, from which tentative hard decisions are subsequently generated. These tentative decisions are then used to control a subsequent, more effective, processing pass through the data, from which more refined hard decisions are generated. The process repeats until sufficiently accurate hard decisions are obtained.

This strategy can be used effectively in multiuser decoding problems involving time-varying intersymbol interference channels. For the purposes of illustration, we focus on the development on such techniques for use with spread-signature CDMA transmission formats specifically, which are well-suited for use in time-varying multipath environments [7] [8]. 
In [7], linear receivers were developed for spread-signature CDMA systems. With such receivers, it was shown that such systems effectively transform the multiuser Rayleigh fading channel into a decoupled set of additive white quasi-Gaussian noise channels. In particular, both the intersymbol and multiple-access interferences are transformed into a second quasi-Gaussian noise source that is effectively white and uncorrelated with the input data stream. This transformation renders these interference terms more benign when hard decisions are generated from the soft symbol estimates using a simple slicer.

As we develop in this part of the paper, further improvements in performance can be achieved not by transforming but by actually canceling some of the interference through an estimatorsubtracter structure that exploits the discrete-alphabet property of the transmitted streams. The particular cancellation strategy we develop here is an effectively linear complexity algorithm like the sequential algorithms (and their DFE variants) developed in the first part of this paper.

It is worth emphasizing at the outset that the scheme we develop can be viewed as an efficient generalization of a variety of stripping (successive decoding) strategies proposed for conventional CDMA systems, such as the multistage algorithm developed by Varanasi and Aazhang [18]. Moreover, in single-user scenarios, where spread-signature CDMA specializes to the class of spread-response precoding algorithms described in [8] [9], the algorithms we develop specialize to an efficient variant of the novel multistage receivers described by Wittneben in [19], into which useful new insights are obtained.

To simplify our development, we restrict our attention to a special case of the basic system model described in Section 2. In particular, in addition to focusing on orthogonal spread-signature CDMA systems with large temporal spreads (i.e., $K \gg L$ ), we also restrict our attention to the case in which the streams $x_{m}[n]$ consist of specifically $N$-PSK (phase-shift keying) symbols. In our development, it will also be convenient to adopt a statistical characterization of the fading process. For this purpose, we consider ergodic Rayleigh fading, so that the $a_{m}[n ; k]$ for different values of $k$ are zero-mean, ergodic, complex-valued, circularly-symmetric, jointly Gaussian sequences. We further restrict our attention to the stationary, uncorrelated scattering scenario, so that the timevarying frequency response of the channel

$$
A_{m}(\omega ; n]=\sum_{k} a_{m}[n ; k] e^{-j \omega}
$$

is stationary in both time $n$ and frequency $\omega$. In terms of this frequency response, the SNR at which the $m$ th user's transmission is received is

$$
\frac{1}{\zeta_{m}}=E\left[\frac{\mathcal{E}_{m}\left|A_{m}\right|^{2}}{\mathcal{N}_{0} \mathcal{W}_{0}}\right]
$$

\subsection{Multipass Receivers}

As discussed in the preceding section, the decoding algorithm we develop is a batch-iterative algorithm involving successive processing of the received data stream. In describing the algorithm, we denote the parameters associated with the processing at the $l$ th iteration using a superscript $l$. The first pass of the algorithm involves applying the linear receiver originally developed for spread-signature CDMA in [7]. We begin by summarizing its salient characteristics, emphasizing aspects that will be important in the development of the processing in subsequent passes of the 
algorithm.

The first decoding pass for the $m$ th user's symbol stream is depicted in Fig. 4. Consistent with our convention, the parameters of all signals and systems involved in this pass have the superscript 1 to reflect that this is the first $(l=1)$ pass of decoding. In this pass, the received data $r[n]$ is first equalized via a linear time-varying filter with kernel $b_{m}^{1}[n ; k]$, producing the sequence

$$
\hat{y}_{m}^{1}[n]=\sum_{k} b_{m}^{1}[n ; k] r[n-k] .
$$

Next, the equalized data $\hat{y}_{m}^{1}[n]$ demodulated from its signature by correlating with the signature $h_{m}[n]$ and downsampling at rate $L$, yielding

$$
\hat{x}_{m}^{1}[n]=\sum_{k} \hat{y}_{m}^{1}[k] h_{m}[k-n L]
$$

Finally, preliminary symbol decisions $\check{x}_{m}^{1}[n]$ are obtained by passing the demodulated data $\hat{x}_{m}^{1}[n]$ through a slicer designed for detection of $N$-PSK symbols in traditional independent additive white Gaussian noise.

Substituting, in turn, (36) and (3) into (37), it follows that the demodulated stream can be expressed in the form

$$
\hat{x}_{m}^{1}[n]=\mu_{m}^{1} x[n]+v_{m}^{1}[n] .
$$

In (38), $\mu_{m}^{1}$ is a constant whose value does not depend on the channel realization but is related to the statistics of the system $c_{i m}^{1}[n ; k]$ formed from cascading the channel $a_{i}[n ; k]$ with the equalizer $b_{m}^{1}[n ; k]$. In particular, $\mu_{m}^{1}$ is defined via the relation

$$
E\left[c_{m i}^{1}[n ; k]\right]=\mu_{m}^{1} \delta[k] \delta[m-i],
$$

where

$$
c_{i m}^{1}[n ; k]=\sum_{l} b_{m}^{1}[n ; l] a_{i}[n-l ; k-l]
$$

Meanwhile, the term $v_{m}^{1}[n]$ in (38) can be expressed in the form

$$
v_{m}^{1}[n]=u_{m}^{1}[n]+\sum_{i=1}^{M} z_{i m}^{1}[n],
$$

where $u_{m}^{1}[n]$ is a noise term and the $z_{i m}^{1}[n]$ are interference terms. In particular,

$$
u_{m}^{1}[n]=\sum_{k} g_{m}^{1}[n ; k] w[n-k]
$$

with

$$
g_{m}^{1}[n ; k]=\sum_{k_{1}} b_{m}^{1}\left[k_{1} ; k_{1}-n+k\right] h_{m}\left[k_{1}-n L\right]
$$


The component $z_{i m}^{1}[n]$ is specifically the interference due to the $i$ th user, i.e.,

$$
z_{i m}^{1}[n]=\sum_{k} f_{i m}^{1}[n ; k] x_{i}[n-k]
$$

where

$$
f_{i m}^{1}[n ; k]=\sum_{k_{1}} \sum_{k_{2}} \tilde{c}_{i m}^{1}\left[k_{1} ; k_{2}\right] h_{m}\left[k_{1}-n L\right] h_{i}\left[k_{1}-n L-k_{2}+k L\right]
$$

with

$$
\tilde{c}_{i m}^{1}[n ; k]=c_{i m}^{1}[n ; k]-E\left[c_{i m}^{1}[n ; k]\right]
$$

As such, $z_{m m}^{1}[n]$ represents the intersymbol interference and the remaining $z_{i m}^{1}[n]$, for $i \neq m$, are multiple-access interference terms.

Under reasonably mild conditions, these noise and interference terms have some convenient characteristics. The specific requirements, which are formally defined and developed in [7], are that the symbol stream be stationary and white, that the channel and equalizer be what is termed sufficiently ergodic, and that the orthogonal signatures be what is termed sufficiently dispersed and partitioned. For our purposes, it suffices to appreciate that these conditions imply, among other properties, that the channel experiences significant time-variation over the effective signature lengths involved. In this case, the statistics of the noise and interference terms do not depend on the particular realization of the channel in effect, only on the ensemble average properties of the channel. In particular, we have ${ }^{2}$

$$
\begin{aligned}
E\left[u_{m}^{1}[n]\right] & =0 \\
\operatorname{cov}\left(u_{m}^{1}[n], u_{m}^{1}[k]\right) & =\mathcal{N}_{0} \mathcal{W}_{0} E\left[\left|B_{m}^{1}\right|^{2}\right] \delta[n-k],
\end{aligned}
$$

where

$$
B_{m}^{1}(\omega ; n]=\sum_{k} b_{m}^{1}[n ; k] e^{-j \omega k}
$$

and

$$
\begin{aligned}
E\left[z_{i m}^{1}[n]\right] & =0 \\
\operatorname{cov}\left(z_{i_{1} m}^{1}[n], z_{i_{2} m}^{1}[k]\right) & =\frac{\mathcal{E}_{i}}{L} \operatorname{var}\left[C_{m}^{1}\right] \delta\left[i_{1}-i_{2}\right] \delta[n-k]
\end{aligned}
$$

where

$$
C_{i m}^{1}(\omega ; n]=\sum_{k} c_{i m}^{1}[n ; k] e^{-j \omega k}
$$

\footnotetext{
${ }^{2}$ Note that within expectations, we omit arguments of the form $(\omega ; n]$ from time-varying frequency responses due to stationarity.
} 
Due to the underlying model, the noise term $u_{m}^{1}[n]$ and multiple-access interference terms $v_{i m}^{1}[n]$, $i \neq m$, are obviously uncorrelated with the symbol stream $x[n]$. However, in this scenario the intersymbol interference term $v_{m m}^{1}[n]$ is also uncorrelated with $x[n]$ [7]. To see this, it suffices to note that as the signature spread $K$ becomes large relative to the channel coherence time, the coefficient $f_{m m}^{1}[n ; 0]$ in (44) that multiplies $x_{m}[n]$ becomes arbitrarily small (in a mean-square sense).

Under these conditions, the second-order characteristics of the system up to the input of the slicer are fully described by the asymptotic signal-to-noise+interference ratio (SNIR)

$$
\gamma_{m}^{1}\left(B_{m}^{1}\right)=\frac{\left|\mu_{m}^{1}\right|^{2} \mathcal{E}_{m}}{\mathcal{N}_{0} \mathcal{W}_{0} E\left[\left|B_{m}^{1}\right|^{2}\right]+\frac{1}{L} \sum_{i=1}^{M} \mathcal{E}_{i} \operatorname{var} C_{i m}^{1}}
$$

Moreover, that the marginal distribution of the interference is asymptotically Gaussian suggests that the symbol error rate performance of the symbol-by-symbol threshold detector for the $N$-PSK stream $x_{m}[n]$ can be reasonably approximated by that given in [20] for the corresponding additive white Gaussian noise channel, i.e.,

$$
P_{m}^{1} \approx 2 \mathcal{Q}\left(\sin \left(\frac{\pi}{N}\right) \sqrt{2 \gamma_{m}^{1}}\right)
$$

where

$$
\mathcal{Q}(v)=\frac{1}{\sqrt{2 \pi}} \int_{v}^{\infty} e^{-t^{2} / 2} d t
$$

In practice, this approximation is a remarkably good one as the simulations in [7] confirm.

From this perspective, it is natural to choose the equalizer so as to maximize the SNIR (53). Provided the fading is sufficiently slow that

$$
C_{i m}^{1}(\omega ; n] \approx B_{m}^{1}(\omega ; n] A_{i}(\omega ; n],
$$

the SNIR-maximizing equalizer has a time-varying frequency response given by [7]

$$
B_{m}^{1}(\omega ; n] \propto \frac{A_{m}^{*}(\omega ; n]}{\mathcal{N}_{0} \mathcal{W}_{0}+\frac{1}{L} \sum_{i=1}^{M} \mathcal{E}_{i}^{l-1}\left|A_{i}(\omega ; n]\right|^{2}},
$$

Note that, interestingly, (56) is also the equalizer that would result from a MMSE criterion (without a causality constraint). In fact, it is possible to verify that for typical forward-link transmission this receiver is effectively equivalent to the receiver developed in Section 3.2 provided sufficient smoothing is accommodated (by, for example, state augmentation) in the estimation in the latter.

On the reverse-link, the performance of the linear MMSE receiver in Section 3.2 is superior to that of the first stage developed above because the latter imposes a single-user detection structure rather than equalizing and demodulating all users jointly before making symbol decisions. As a result, the above system is interference-limited as the results in [7] reflect-the bit-error rate does 
not go to zero with increasing SNR - making it vulnerable to near-far effects [16].

\subsubsection{Iterative Interference Estimation-Subtraction}

Performance on both forward and reverse links is improved by making use of these preliminary symbol decisions $\breve{x}_{m}^{1}[n]$ in subsequent processing passes of the received data $r[n]$. The iterative interference suppression strategy we develop in this section has the following form. Using the receiver of Fig. 4, we obtain preliminary decisions $\breve{x}_{m}^{1}[n]$ for each of the $M$ different symbol streams at the receiver. In turn, by subsequent processing of these decisions we generate refined estimates $\hat{z}_{i m}^{2}[n]$ of the interference terms in (41). These estimates are then effectively subtracted from an appropriately equalized and demodulated version of the data to produce $\hat{x}_{m}^{2}[n]$, from which more reliable symbol decisions $\breve{x}_{m}^{2}[n]$ are obtained from the slicer. This process is then repeated, exploiting the decisions $\breve{x}_{m}^{2}[n]$ to generate further refined interference estimates $\hat{z}_{i m}^{3}[n]$, etc. A detailed development follows.

The interference canceler in the $l$ th pass $(l \geq 2)$ takes the form depicted in Fig. 5 . In particular, we subtract an appropriately constructed estimate $\hat{z}_{m}^{l}[n]$ of the total intersymbol and multipleaccess interference from the equalized and demodulated version of the data to produce $\hat{x}_{m}^{l}[n]$, i.e., [cf. (36)-(37)]

$$
\hat{x}_{m}^{l}[n]=\sum_{k} \hat{y}_{m}^{l}[k] h_{m}[k-n L]-\hat{z}_{m}^{l}[n]
$$

where

$$
\hat{y}_{m}^{l}[n]=\sum_{k} b_{m}^{l}[n ; k] r[n-k]
$$

It is important to emphasize that in each pass we allow the equalizer $b_{m}^{l}[n ; k]$, whose time-varying frequency response we denote by

$$
B_{m}^{l}(\omega ; n]=\sum_{k} b_{m}^{l}[n ; k] e^{-j \omega k},
$$

to differ from that used in previous processing passes (including the first pass); this flexibility will prove important.

The associated interference estimate $\hat{z}_{m}^{l}[n]$ used in (57) is a linear combination of estimates of the constituent interferences, i.e.,

$$
\hat{z}_{m}^{l}[n]=\sum_{i=1}^{M} \alpha_{i m}^{l} \hat{z}_{i m}^{l}[n],
$$

where the $\alpha_{i m}^{l}$ are suitably designed weights. The interference estimates $\hat{z}_{i m}^{l}[n]$ for this $l$ th pass are in turn generated in the manner depicted in Fig. 6: the symbol decisions from the previous pass are re-modulated onto the appropriate signature, processed by a noise-free replica of the channel, then demodulated by a replica of the receiver with the equalizer $b_{m}^{l}[n ; k]$ used in the canceler $(57)$. The lower path in Fig. 6 eliminates the symbol of interest from the intersymbol interference estimate, and obviously doesn't arise in the estimation of multiple-access interference $(i \neq m)$. 
Analogous to notation we used in the first pass, we use $c_{i m}^{l}[n ; k]$ to denote the kernel of the system formed from the cascade of the equalizer $b_{m}^{l}[n ; k]$ with the channel corresponding to $a_{i}[n ; k]$, i.e.,

$$
c_{i m}^{l}[n ; k]=\sum_{k_{1}} b_{m}^{l}\left[n ; k_{1}\right] a_{i}\left[n-k_{1} ; k-k_{1}\right]
$$

and use

$$
C_{i m}^{l}(\omega ; n]=\sum_{k} c_{i m}^{l}[n ; k] e^{-j \omega k}
$$

to denote associated time-varying frequency response. The parameter $\mu_{m}^{l}$ in the lower path of Fig. 6 is related to the mean of this cascade channel. In particular, it is defined via the relation

$$
E\left[C_{i m}^{l}(\omega ; n]\right]=\mu_{m}^{l} \delta[m-i]
$$

As a result, the interference estimate can be expressed in the form [cf. (44)-(46)]

$$
\hat{z}_{i m}^{l}[n]=\sum_{k} f_{i m}^{l}[n ; k] \check{x}_{i}^{l-1}[n-k]
$$

where

$$
f_{i m}^{l}[n ; k]=\sum_{k_{1}} \sum_{k_{2}} \tilde{c}_{i m}^{l}\left[k_{1} ; k_{2}\right] h_{m}\left[k_{1}-n L\right] h_{i}\left[k_{1}-n L-k_{2}+k L\right]
$$

with

$$
\tilde{c}_{i m}^{l}[n ; k]=c_{i m}^{l}[n ; k]-E\left[c_{i m}^{l}[n ; k]\right] .
$$

With the above interference estimation-subtraction structure, the input to the subsequent slicer in Fig. 5 can be expressed in the form

$$
\hat{x}_{m}^{l}[n]=\mu_{m}^{l} x_{m}[n]+v_{m}^{l}[n] .
$$

Analogous to (41)-(43), the additive distortion $v_{m}^{l}[n]$ in (67) can be expressed in the form

$$
v_{m}^{l}[n]=u_{m}^{l}[n]+\sum_{i=1}^{M} \tilde{z}_{i m}^{l}[n],
$$

where $u_{m}^{l}[n]$ is the noise term

$$
u_{m}^{l}[n]=\sum_{k} g_{m}^{l}[n ; k] w[n-k]
$$


with

$$
g_{m}^{l}[n ; k]=\sum_{k_{1}} b_{m}^{l}\left[k_{1} ; k_{1}-n+k\right] h_{m}\left[k_{1}-n L\right],
$$

and where the $\tilde{z}_{i m}^{l}[n]$ are residual interference terms

$$
\tilde{z}_{i m}^{l}[n]=z_{i m}^{l}[n]-\alpha_{i m}^{l} \hat{z}_{i m}^{l}[n]
$$

in which [cf. (64)]

$$
z_{i m}^{l}[n]=\sum_{k} f_{i m}^{l}[n ; k] x_{i}[n-k] .
$$

\subsection{Receiver Optimization}

Not surprisingly, the choice of both the equalizer coefficients $b_{m}^{l}[n ; k]$ and canceler weights $\alpha_{i m}^{l}$ in each pass $l$ of receiver processing strongly affect the quality and convergence characteristics of the symbol decisions produced in each pass. One rather natural way to select these parameters is so that in each pass the slicer input SNIR is maximized.

Assuming, as was true in the first processing pass, that for any particular realization of the channel the noise terms $u_{m}^{l}[n]$ and residual interference terms $\tilde{z}_{i m}^{l}[n]$ in (68) are each asymptotically zero-mean, stationary, white, uncorrelated with one another, and uncorrelated with the symbol stream $x_{m}[n]$, then the SNIR at the input to the slicer in the $l$ th processing pass can be expressed in the form

$$
\gamma_{m}^{l}\left(b_{m}^{l}, \alpha_{1 m}^{l}, \alpha_{2 m}^{l}, \ldots, \alpha_{M m}^{l}\right)=\frac{\left|\mu_{m}^{l}\right|^{2} \mathcal{E}_{m}}{\operatorname{var} u_{m}^{l}+\sum_{i=1}^{M} \operatorname{var} z_{i m}^{l}} .
$$

It then remains to express the quantities in (73) in terms of the parameters to be optimized.

Expressing the first denominator term in (73) in the desired form is straightforward in this scenario:

$$
\operatorname{var} u_{m}^{l}[n]=\mathcal{N}_{0} \mathcal{W}_{0} E\left[\left|B_{m}^{l}\right|^{2}\right]
$$

To obtain the remaining denominator terms, we assume that

$$
\operatorname{var} \hat{z}_{i m}^{l}[n] \approx \operatorname{var} z_{i m}^{l}[n]=\mathcal{E}_{i} \sum_{k}\left|f_{i m}^{l}[n ; k]\right|^{2}=\frac{\mathcal{E}_{i}}{L} \operatorname{var} C_{m}^{l},
$$

which is valid at least at high SNR, and that the correlation between the actual symbols and intermediate decisions is one of the form

$$
E\left[x_{i}^{*}[n] \check{x}_{m}^{l}[k]\right] \approx \rho_{m}^{l} \mathcal{E}_{m} \delta[n-k] \delta[i-m],
$$


where the $\rho_{m}^{l}$ are correlation coefficients that can be calculated. In this case, using the approximations (75) and (76) with (64) and (72) in (71), we obtain

$$
\frac{\operatorname{var} \tilde{z}_{i m}^{l}}{\operatorname{var} z_{i m}^{l}} \approx 1+\left|\alpha_{i m}^{l}\right|^{2}-2 \operatorname{Re}\left\{\alpha_{i m}^{l} \rho_{i}^{l-1}\right\}=1+\left|\rho_{i}^{l-1}\right|^{2}+\left|\alpha_{i m}^{l}-\left(\rho_{i}^{l-1}\right)^{*}\right|^{2}
$$

In turn, substituting the right-hand side of (75) into (77), it is straightforward to verify that

$$
\operatorname{var} \tilde{z}_{i m}^{l} \geq \frac{\tilde{\mathcal{E}}_{i}^{l}}{L} \operatorname{var} C_{i m}^{l}
$$

where

$$
\tilde{\mathcal{E}}_{i}^{l-1}=\mathcal{E}_{i}\left(1-\left|\rho_{i}^{l-1}\right|^{2}\right)
$$

with equality if and only if

$$
\alpha_{i m}^{l}=\left(\rho_{i}^{l-1}\right)^{*}
$$

Hence, the maximum achievable SNIR is obtained when the weights $\alpha_{i m}^{l}$ are chosen according to (80).

Using (78), (74), and (63) in (73) yields an SNIR in terms of the optimized weights (80) of the form

$$
\gamma_{m}^{l}\left(B_{m}^{l}\right)=\frac{\left|E\left[C_{i m}^{l}\right]\right|^{2} \mathcal{E}_{m}}{\mathcal{N}_{0} \mathcal{W}_{0} E\left[\left|B_{m}^{l}\right|^{2}\right]+\frac{1}{L} \sum_{i=1}^{M} \tilde{\mathcal{E}}_{i}^{l-1} \operatorname{var} C_{i m}^{l}} .
$$

Note that $(53)$ can be viewed as a special case of (81) when we adopt the convention that $\rho_{m}^{0} \triangleq 0$.

Finally, assuming sufficiently slow fading that in each pass $l$ the approximation

$$
C_{i m}^{l}(\omega ; n] \approx B_{m}^{l}(\omega ; n] A_{i}(\omega ; n]
$$

is valid, then (81) can then be further expanded as

$$
\gamma_{m}^{l}\left(B_{m}^{l}\right)=\frac{\left|E\left[A_{m} B_{m}^{l}\right]\right|^{2} \mathcal{E}_{m}}{\mathcal{N}_{0} \mathcal{W}_{0} E\left[\left|B_{m}^{l}\right|^{2}\right]+\frac{1}{L} \sum_{i=1}^{M} \tilde{\mathcal{E}}_{i}^{l-1} \operatorname{var}\left[A_{i} B_{i}^{l}\right]}
$$

In this form, it is straightforward to optimize the SNIR $\gamma_{m}^{l}$ over the choice of equalizer frequency response $B_{m}^{l}(\omega ; n]$. We consider forward- and reverse-link scenarios separately.

In the forward-link scenario, all $x_{m}[n]$ pass through the same channel [see (11)]. As a result we can drop the subscripts from our notation for the channel, equalizer, and cascade frequency responses, using, respectively, $A(\omega ; n], B^{l}(\omega ; n]$, and $C^{l}(\omega ; n]$ for all $m$. In this case, (83) specializes 
to

$$
\gamma_{m}^{l}\left(B_{m}^{l}\right)=\frac{\left|E\left[A B^{l}\right]\right|^{2} \mathcal{E}_{m}}{\mathcal{N}_{0} \mathcal{W}_{0} E\left[\left|B^{l}\right|^{2}\right]+\overline{\mathcal{E}}^{l-1} \operatorname{var}\left[A B^{l}\right]},
$$

where

$$
\overline{\mathcal{E}}^{l}=\frac{1}{L} \sum_{i=1}^{M} \tilde{\mathcal{E}}_{i}^{l}
$$

With $\gamma_{m}^{l}$ in the form (84), a special case of Lemmas 1 and 2 from [7] can be applied to obtain that

$$
\gamma_{m}^{l}\left(B^{l}\right) \leq \frac{\bar{\zeta}^{l-1}}{\zeta_{m}}\left[\frac{1}{\bar{\zeta}^{l-1} \exp \left(\bar{\zeta}^{l-1}\right) E_{1}\left(\bar{\zeta}^{l-1}\right)}-1\right]
$$

with equality if and only if

$$
B^{l}(\omega ; n] \propto \frac{A_{m}^{*}(\omega ; n]}{\mathcal{N}_{0} \mathcal{W}_{0}+\overline{\mathcal{E}}^{l-1}|A(\omega ; n]|^{2}} .
$$

In $(86)$,

$$
\frac{1}{\bar{\zeta}^{l}}=E\left[\frac{\overline{\mathcal{E}}^{l}|A|^{2}}{\mathcal{N}_{0} \mathcal{W}_{0}}\right]=\frac{1}{L} \sum_{i=1}^{M} \frac{1-\left|\rho_{i}^{l}\right|^{2}}{\zeta_{i}},
$$

with $\zeta_{m}$ as given in (35), and $E_{1}(\cdot)$ denotes the exponential integral, i.e.,

$$
E_{1}(v)=\int_{v}^{\infty} \frac{e^{-t}}{t} d t
$$

For reverse-link transmission, the general cases of Lemmas 1 and 2 from [7] can be applied to show that (83) satisfies

$$
\gamma_{m}^{l}\left(B_{m}^{l}\right) \leq \frac{L}{1-\left|\rho_{m}^{l-1}\right|^{2}}\left(\frac{1}{\beta_{m}^{l-1}}-1\right)
$$

with equality if and only if

$$
B_{m}^{l}(\omega ; n] \propto \frac{A_{m}^{*}(\omega ; n]}{\mathcal{N}_{0} \mathcal{W}_{0}+\frac{1}{L} \sum_{i=1}^{M} \tilde{\mathcal{E}}_{i}^{l-1}\left|A_{i}(\omega ; n]\right|^{2}} .
$$


In $(89)$,

$$
\beta_{m}^{l}=\frac{M-1}{M}+\frac{\left(L \tilde{\zeta}_{m}^{l}\right)^{M}}{M !}\left[(-1)^{M+1} e^{L \tilde{\zeta}_{m}^{l}} E_{1}\left(L \tilde{\zeta}_{m}^{l}\right)+\sum_{k=0}^{M-2}(-1)^{M-k} \frac{k !}{\left(L \tilde{\zeta}_{m}^{l}\right)^{k+1}}\right]
$$

with

$$
\frac{1}{\tilde{\zeta}_{m}^{l}}=E\left[\frac{\tilde{\mathcal{E}}_{m}^{l}\left|A_{m}\right|^{2}}{\mathcal{N}_{0} \mathcal{W}_{0}}\right]=\frac{1-\left|\rho_{m}^{l}\right|^{2}}{\zeta_{m}}
$$

where again $\zeta_{m}$ is as given in (35).

Hence (87) and (90) are the optimum equalizers for the forward and reverse links, respectively, and the associated slicer input SNIR's that are attained with these equalizers are given by the corresponding right-hand sides of (86) and (89). Implementing each of these equalizers requires explicit knowledge of the set of correlation coefficients $\rho_{m}^{l}$ that characterize the quality of the symbol decisions produced at the output of the slicer in each processing pass. Conveniently, these coefficients can be computed sequentially using an efficient recursive algorithm, as we now develop.

\subsection{Weight and Performance Calculation Recursion}

To obtain a suitable recursion, we first express the desired correlation coefficients $\rho_{m}^{l}$ in terms of the symbol error probability $P_{m}^{l}$ at the output of the corresponding slicer. To complete the recursion, we then determine an expression for the symbol error probability $P_{m}^{l}$ as a function of the corresponding correlation coefficients $\rho_{m}^{l-1}$ associated with the symbol decisions from the previous processing pass.

We note in advance that this not only yields a recursive algorithm for computing the correlation coefficients, but also one for predicting the error probabilities of the symbol decisions generated in each pass of the algorithm. This information is useful both in determining asymptotic performance limits and in estimating a priori the number of decoding iterations required in practice, as we will discuss later.

To express the coefficient $\rho_{m}^{l}$ in terms of $P_{m}^{l}$, we begin by writing the error at the output of the associated slicer in the form

$$
e_{m}^{l}[n]=\check{x}_{m}^{l}[n]-x_{m}[n],
$$

and note that when $N>2$ and $x_{m}[n]=\sqrt{\mathcal{E}_{m}}$, we have the approximation

$$
\begin{aligned}
\operatorname{Pr}\left[\frac{e_{m}^{l}[n]}{\sqrt{\mathcal{E}_{m}}}=-2 \sin ^{2}\left(\frac{\pi}{N}\right)+j 2 \sin \left(\frac{\pi}{N}\right) \cos \left(\frac{\pi}{N}\right)\right]= \\
\operatorname{Pr}\left[\frac{e_{m}^{l}[n]}{\sqrt{\mathcal{E}_{m}}}=-2 \sin ^{2}\left(\frac{\pi}{N}\right)-j 2 \sin \left(\frac{\pi}{N}\right) \cos \left(\frac{\pi}{N}\right)\right] \approx \frac{P_{m}^{l}}{2} .
\end{aligned}
$$

Thus, exploiting, in turn, symmetry and (94) we obtain

$$
E\left[x_{m}^{*}[n] e_{m}^{l}[n]\right]=E\left[x_{m}^{*}[n] e_{m}^{l}[n] \mid x_{m}[n]=\sqrt{\mathcal{E}_{m}}\right] \approx-2 \sin ^{2}\left(\frac{\pi}{N}\right) \mathcal{E}_{m} P_{m}^{l}
$$


Finally, using (95) with (76), we obtain, for $l \geq 1$, our desired expression

$$
\rho_{m}^{l}=\frac{E\left[\left|x_{m}[n]\right|^{2}\right]+E\left[x_{m}^{*}[n] e_{m}^{l}[n]\right]}{\mathcal{E}_{m}} \approx 1-2 \sin ^{2}\left(\frac{\pi}{N}\right) \mathcal{E}_{m} P_{m}^{l} .
$$

Note that (96) implies that, as we would expect, for constellations with such symmetry the correlation coefficient is real: $\rho_{m}^{l}=\left(\rho_{m}^{l}\right)^{*}$.

It is similarly straightforward to develop an expression for $P_{m}^{l}$ in terms of $\rho_{m}^{l-1}$. We begin by observing that we can express $P_{m}^{l}$ in terms of $\gamma_{m}^{l}$ using an approximation analogous to that used to obtain (54). In particular, by neglecting any statistical dependence between $v_{m}^{l}[n]$ and $x[n]$ in (67), and treating $v_{m}^{l}[n]$ as a stationary, white, Gaussian process, the $N$-PSK symbol error rate for the decisions produced by the slicer can be expressed as

$$
P_{m}^{l} \approx 2 \mathcal{Q}\left(\sin \left(\frac{\pi}{N}\right) \sqrt{2 \gamma_{m}^{l}}\right)
$$

Finally, to complete the mapping from $\rho_{m}^{l-1}$ to $P_{m}^{l}$, we substitute the right-hand side of either (86) or (89) into (97), depending on whether the scenario of interest is forward- or reverse-link transmission, respectively.

We conclude this section with a summary of the recursion for determining the correlation coefficients required in the equalizer and for predicting the symbol error rate performance as a function of the number of iterations used:

1. Set $\rho_{m}^{0}=0$ and let $l=1$.

2. For each $m$, compute the SNIR $\gamma_{m}^{l}$ at the slicer input on the $l$ th decoding pass from the correlation coefficients $\rho_{m}^{l-1}$ and channel SNR via the right-hand side of either (86) or (89) for forward- or reverse-link transmission, respectively. Compute the symbol error probability $P_{m}^{l}$ for the slicer output from the slicer input SNIR $\gamma_{m}^{l}$ via (97).

3. For each $m$, from the symbol error probabilities $P_{m}^{l}$ produced in Step 2 compute the correlation coefficients $\rho_{m}^{l}$. via $(96)$.

4. Increment $l$ and go to step 2 .

\subsection{Performance Characteristics}

In this section, we develop some properties of the performance predictions, and compare these predictions to the results of Monte Carlo experiments. For the purposes of illustration, in both forward- and reverse-link transmission we restrict our attention to the scenario in which $\zeta_{m}=\zeta$ for all $m$, in which case we may omit the subscripts from our notation for the correlation coefficients, SNIR's, and symbol error probabilities-yielding, respectively, $\rho^{l}, \gamma^{l}$, and $P^{l}$. Note that on the reverse link, this corresponds to the case in which power control is employed.

The characteristics of the performance predictions are best understood graphically, as we now illustrate in the case of the forward link with QPSK $(N=4), M=8$ users, no excess bandwidth $(L=M)$, and maximally-spread signatures. 
For a given received SNR level $1 / \zeta$, the relationship between $\rho^{l-1}$ and $P^{l}$ corresponding to Step 2 of the above procedure is one of the form

$$
P^{l}=F\left(\zeta, \rho^{l-1}\right),
$$

where the function $F(\cdot, \cdot)$ has the property that it is decreasing with both SNR $1 / \zeta$ and correlation $\rho^{l}$, as can be verified by substituting the right-hand side of (86) into (97). The solid curves of Fig. 7 show the function $F(\zeta, \rho)$ plotted as a function of $1 /(1-\rho)$ on a log-log scale for several values of $1 / \zeta$.

Meanwhile, the mapping (96) between $P^{l}$ and $\rho^{l}$ in Step 3 takes the form

$$
P^{l}=G\left(\rho^{l}\right)
$$

where the function $G(\cdot)$ is also decreasing with $\rho^{l}$. The function $G(\rho)$ is linear as a function $1 /(1-\rho)$ when plotted on a log-log scale, as depicted in Fig. 7 via the dashed line.

For a given operating SNR, the progression of symbol error probabilities and correlation coefficients for successive processing passes is obtained by iterating between the associated solid curve and the dashed line in Fig. 7. In particular, the value of the solid curve at $\rho=0$ determines the symbol error probability $P^{1}$ at the slicer output after the first stage of processing. The correlation coefficient $\rho^{1}$ is then obtained as the value of $\rho$ at which the dashed line takes on the value $P^{1}$. The symbol error probability $P^{2}$ after the second stage is then the height of the solid curve at $\rho=\rho^{1}$, and the process repeats.

Graphically, this locus of operating points is obtained by a moving horizontally from the solid curve to the dashed curve, and vertically from the dashed curve back down to the solid curve. This corresponds to a descending a staircase where there is one such step for each processing pass. In Fig. 7, the sequence of operating points obtained in this manner are depicted via the $\circ$ symbols along each solid curve.

From this analysis, it is apparent that the sequence of symbol error probabilities $P^{1}, P^{2}, \ldots$ obtained by this iterative algorithm is both monotonically decreasing and convergent. This implies that additional processing passes are always beneficial in terms of reducing error rate performance. The steady-state symbol error probability $P^{\infty}$ for an SNR of $1 / \zeta$ follows immediately as the unique solution to the equation

$$
P^{\infty}=F\left(\zeta, G^{-1}\left(P^{\infty}\right)\right)
$$

where $G^{-1}(\cdot)$ is the inverse of $G(\cdot)$, i.e., [cf. (99)], $\rho^{l}=G^{-1}\left(P^{l}\right)$. This steady-state error probability corresponds to the intersection of the dashed line and appropriate solid curve in Fig. 7.

As Fig. 7 reflects, much of the steady-state performance is achieved with relatively few iterations, after which there are strongly diminishing returns to additional processing passes. And while these performance characteristics are based on theoretical predictions, in practice they appear to be quite accurate for this forward-link case. For example, the sequence of experimentally obtained operating points are identified by the $*$ symbols in Fig. 7 . In these experiments, symbol error rates were measured empirically, and the appropriate correlation coefficients were computed numerically as sample-averages from streams generated in simulations.

The asymptotic efficiency of the iterative receiver can also be inferred from Fig. 7. To see this, note from (86) and (89) that in both forward- and reverse-link scenarios $\gamma_{m}^{l+1} \rightarrow 1 / \zeta_{m}$ when $\rho_{i}^{l} \rightarrow 1$ 
for all $i$, which when substituted into (97) yields the performance of the classical additive white Gaussian noise channel. Thus, each of the solid curves in Fig. 7 approach this performance level from above with increasing $\rho$. Since the dashed line intersects the solid curve at larger values of $\rho$ as the SNR $1 / \zeta$ increases, this implies that the performance of the classical additive white Gaussian noise channel is approached at high SNR.

This asymptotically perfect interference cancellation behavior is more directly apparent in Fig. 8, where error rate is plotted as a function of SNR for different numbers of receiver processing passes. As we also see from this figure, on the order of only $(l \leq 5)$ iterations of the decoding algorithm are required to converge to typical target bit error rates. This is significant since the amount of computation is directly proportional to the number of iterations required. We emphasize that the complexity is substantially lower than maximum likelihood sequence detection.

Performance predictions of the type developed above can be obtained in a similar manner for reverse link scenarios. As an illustration, Fig. 9 depicts performance as a function of the number of decoding iterations in a power-controlled system with $M=2$ users, no bandwidth expansion $(L=M)$, and QPSK $(N=4)$ symbols. The corresponding plots of predicted performance as a function of SNR are shown in Fig. 10. As these figures reflect, a modest number of iterations generally suffices for convergence in this reverse-link scenario as well. It is important to point out, however, that when large numbers of users are involved, the predictions tend to be somewhat overly optimistic even at high SNR. This suggests that some of the approximations used in the system optimization and analysis in preceding sections may be less appropriate in such cases.

\section{Concluding Remarks}

The algorithms developed in this paper are representative of several emerging next-generation receiver structures for CDMA systems. However, while promising, many issues remain to be explored and addressed before receivers of this type can be considered for practical implementation.

In terms of the iterative decoding, one obviously important area for further research involves further improving the performance of the multipass receiver through algorithmic enhancements and developing more accurate predictions of the resulting performance, particularly on the reverse-link with typical numbers of users.

Other issues that warrant further investigation include robustness of the iterative decoding algorithm. Preliminary simulations suggest that the performance achieved with each pass is quite sensitive to precise specification of the correlation coefficients. Quantifying this sensitivity and developing versions of the algorithm that are inherently more robust to correlation coefficient modeling and quantization errors is an important area for further work.

In addition, exploring the impact of using the channel estimates available in practice in the equalizer construction rather than perfect channel knowledge is important in terms of practical application. From this perspective, extensions of the kinds of robust equalization techniques developed in [21] for spread-response precoding systems are likely to prove valuable. Also of interest would be blind variants of the algorithms in this paper that don't require training data or pilot tones. The recent work of, among others, Poor and Wang [22] has shown that blind algorithms for such problems are feasible.

As suggested in the introduction, both the batch-iterative and earlier sequential decisionfeedback decoding algorithms developed in this paper can, in principle, be used directly in the receivers of multiuser systems exploiting transmitter antenna diversity - such as those developed 
in [10] — to enhance performance. As such, exploring their use in this context also warrants further research.

Finally, important new insights are likely to arise from an in-depth exploration the relationships between the two classes of decoding algorithms developed in this paper. More generally, a great deal of interesting and important research remains to be done on interrelating the wide range of

algorithms currently under development in the community within this active and exciting area of research.

\section{References}

[1] R. Lupas and S. Verdú, "Linear multiuser detectors for synchronous code-division multipleaccess channels," IEEE Trans. Inform. Theory, vol. 35, pp. 123-136, Jan. 1989.

[2] R. Lupas and S. Verdú, "Near-far resistance of multiuser detectors in asynchronous channels," IEEE Trans. Commun., vol. 38, pp. 496-508, Apr. 1990.

[3] U. Madhow and M. L. Honig, "MMSE interference suppression for direct-sequence spreadspectrum CDMA," IEEE Trans. Commun., vol. 42, pp. 3178-3188, Dec. 1994.

[4] M. Honig, U. Madhow, and S. Verdú, "Blind adaptive multiuser detection," IEEE Trans. Inform. Theory, vol. 41, pp. 944-960, July 1995.

[5] M. L. Honig and H. V. Poor, "Adaptive interference suppression in wireless systems," in Wireless Communications: Signal Processing Perspectives (H. V. Poor and G. W. Wornell, eds.), ch. 2, Upper Saddle River, NJ: Prentice-Hall, 1998.

[6] G. W. Wornell, "Efficient linear diversity techniques for fading channels," in Wireless Communications: Signal Processing Perspectives (H. V. Poor and G. W. Wornell, eds.), ch. 1, Upper Saddle River, NJ: Prentice-Hall, 1998.

[7] G. W. Wornell, "Spread-signature CDMA: Efficient multiuser communication in the presence of fading," IEEE Trans. Inform. Theory, vol. 41, pp. 1418-1438, Sept. 1995.

[8] G. W. Wornell, "Emerging applications of multirate systems and wavelets in digital communications," Proc. IEEE, vol. 84, Apr. 1996.

[9] G. W. Wornell, "Spread-response precoding for communication over fading channels," IEEE Trans. Inform. Theory, vol. 42, pp. 488-501, Mar. 1996.

[10] G. W. Wornell and M. D. Trott, "Efficient signal processing techniques for exploiting transmit diversity on fading channels," IEEE Trans. Signal Processing, vol. 45, pp. 191-205, Jan. 1997.

[11] B. D. O. Anderson and J. B. Moore, Optimal Filtering. Englewood Cliffs, NJ: Prentice-Hall, 1979.

[12] S. Haykin, Adaptive Filter Theory. Englewood Cliffs, NJ: Prentice-Hall, 1986. 
[13] H. C. Papadopoulos, "Equalization for multiuser wireless systems," in Wireless Communications: Signal Processing Perspectives (H. V. Poor and G. W. Wornell, eds.), ch. 3, Upper Saddle River, NJ: Prentice-Hall, 1998.

[14] A. Duel-Hallen, "Equalizers for multiple input/multiple output channels and PAM systems with cyclostationary input sequences," IEEE J. Select. Areas Commun., vol. 10, pp. 630-639, Apr. 1992.

[15] G. D. Forney, Jr., "Maximum-likelihood sequence estimation of digital sequences in the presence of intersymbol interference," IEEE Trans. Inform. Theory, vol. IT-18, pp. 363-378, May 1972 .

[16] S. Verdú, "Minimum probability of error for asynchronous Gaussian multiple-access channels," IEEE Trans. Inform. Theory, vol. IT-32, pp. 85-96, Jan. 1986.

[17] J. M. Cioffi, G. P. Dudevoir, M. V. Eyuboglu, and G. D. Forney, Jr., "MMSE decision-feedback equalizers and coding-Parts I and II," IEEE Trans. Commun., vol. 43, pp. 2582-2604, Oct. 1995.

[18] M. K. Varanasi and B. Aazhang, "Multistage detection in asynchronous code-division multipleaccess communications," IEEE Trans. Commun., vol. 38, pp. 509-519, Apr. 1990.

[19] A. Wittneben, "A novel bandwidth efficient analog coding/decoding scheme for data transmission over fading channels," in Proc. IEEE GLOBECOM, 1994.

[20] J. G. Proakis, Digital Communications. New York, NY: McGraw-Hill, 2nd ed., 1989.

[21] J. N. Laneman, "Channel estimation and equalization for spread-response precoding systems in fading environments," Master's thesis, M. I. T., Cambridge, MA, June 1997.

[22] H. V. Poor and X. Wang, "Blind adaptive joint suppression of MAI and ISI in CDMA channels," in Proc. Asilomar Conf. Signals, Systems, Computers, 1997. 


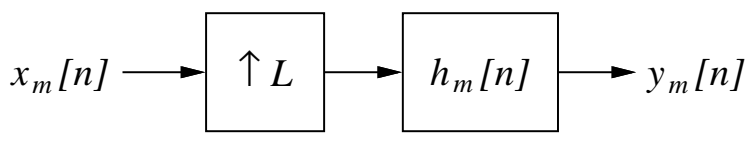

Figure 1: Modulation of the the $m$ th user's coded symbol stream $x_{m}[n]$ onto a signature sequence $h_{m}[n]$ for transmission.

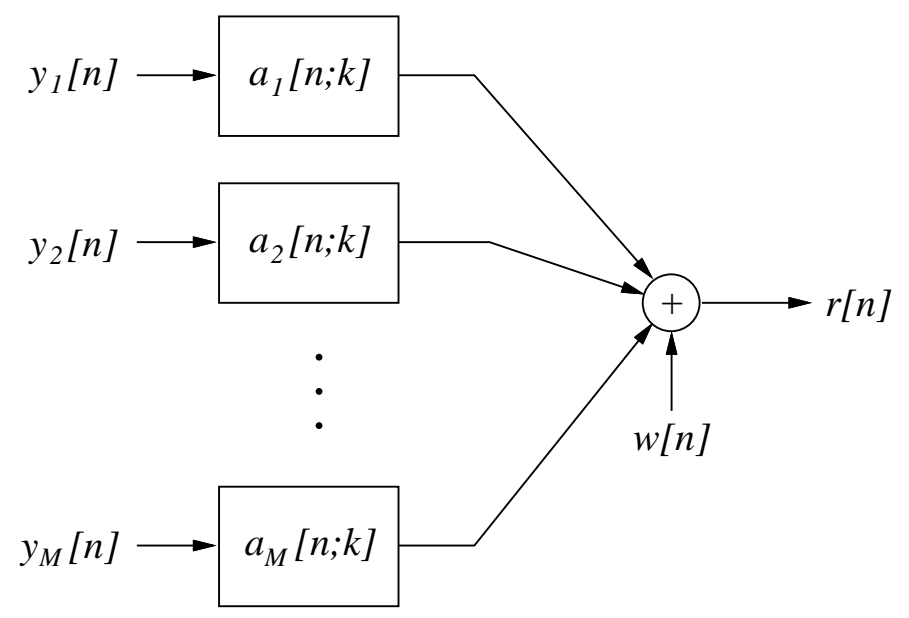

Figure 2: General multiuser intersymbol interference channel model, where $a_{m}[n]$ denotes the unitsample response corresponding to the mth user. 

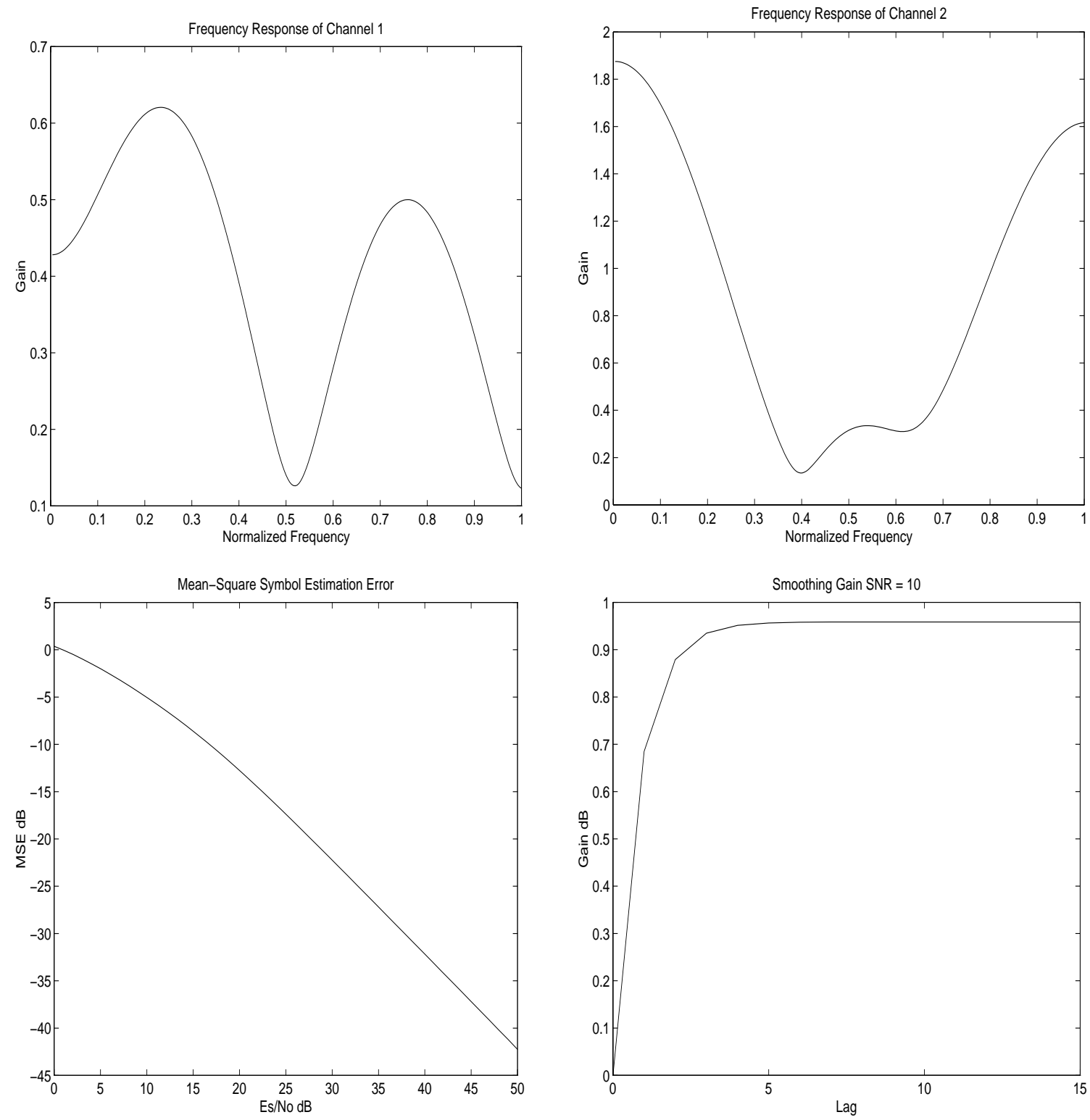

Figure 3: Performance of the linear MMSE multiuser equalizer in a $M=2$ user time-invariant scenario.

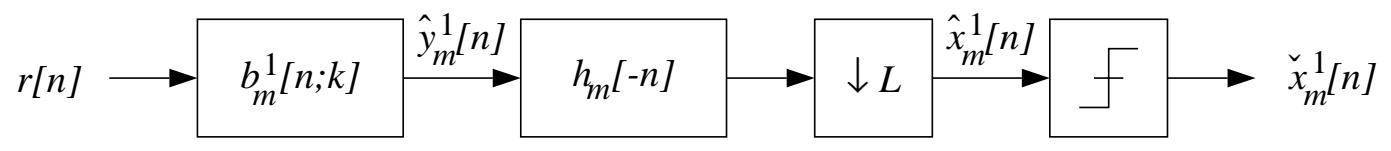

Figure 4: Processing in the first pass of the iterative receiver. 


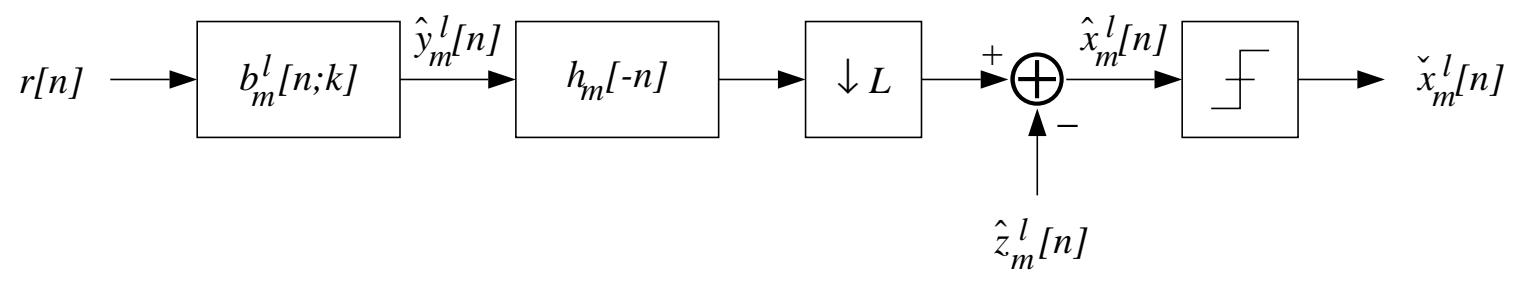

Figure 5: Interference cancellation in the lth decoding pass.

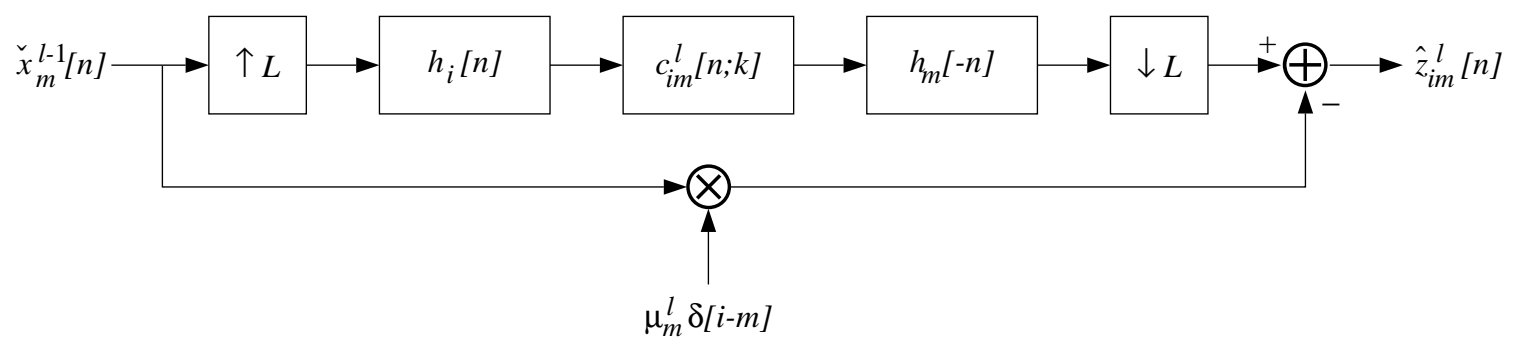

Figure 6: Interference estimation for the lth decoding pass. 


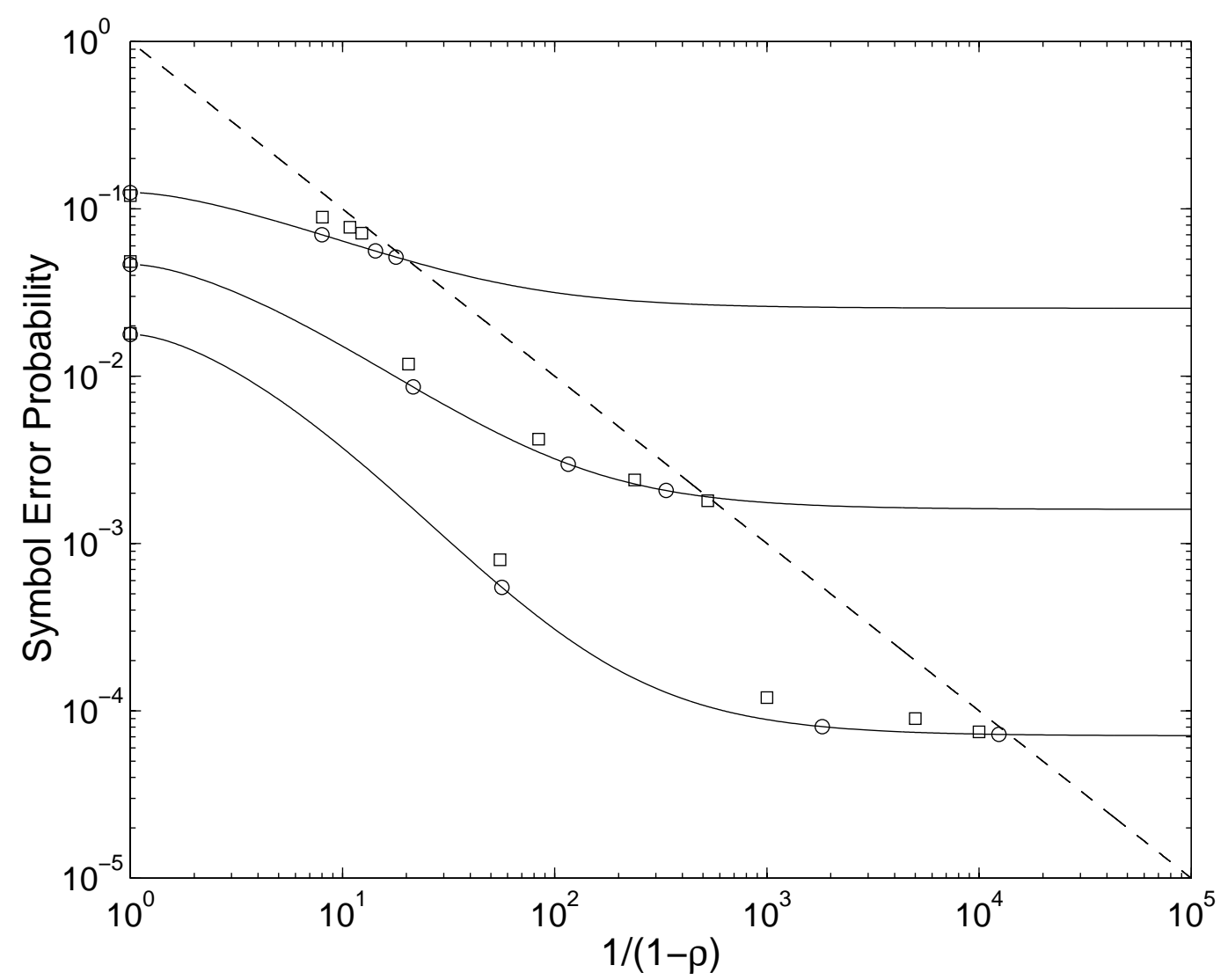

Figure 7: Iterative receiver performance on the forward link. The successively lower solid curves plot QPSK symbol error rate as a function of the correlation coefficient $\rho$ for SNRs of 7, 10, and 12 dB. Along each curve, circles (O) identify the theoretically predicted decreasing error rates achieved with $l=1,2, \ldots$ decoding passes, and the intersections with the dashed line are the steady-state values $(l \rightarrow \infty)$. The associated experimentally obtained values are depicted using squares $(\square)$. 


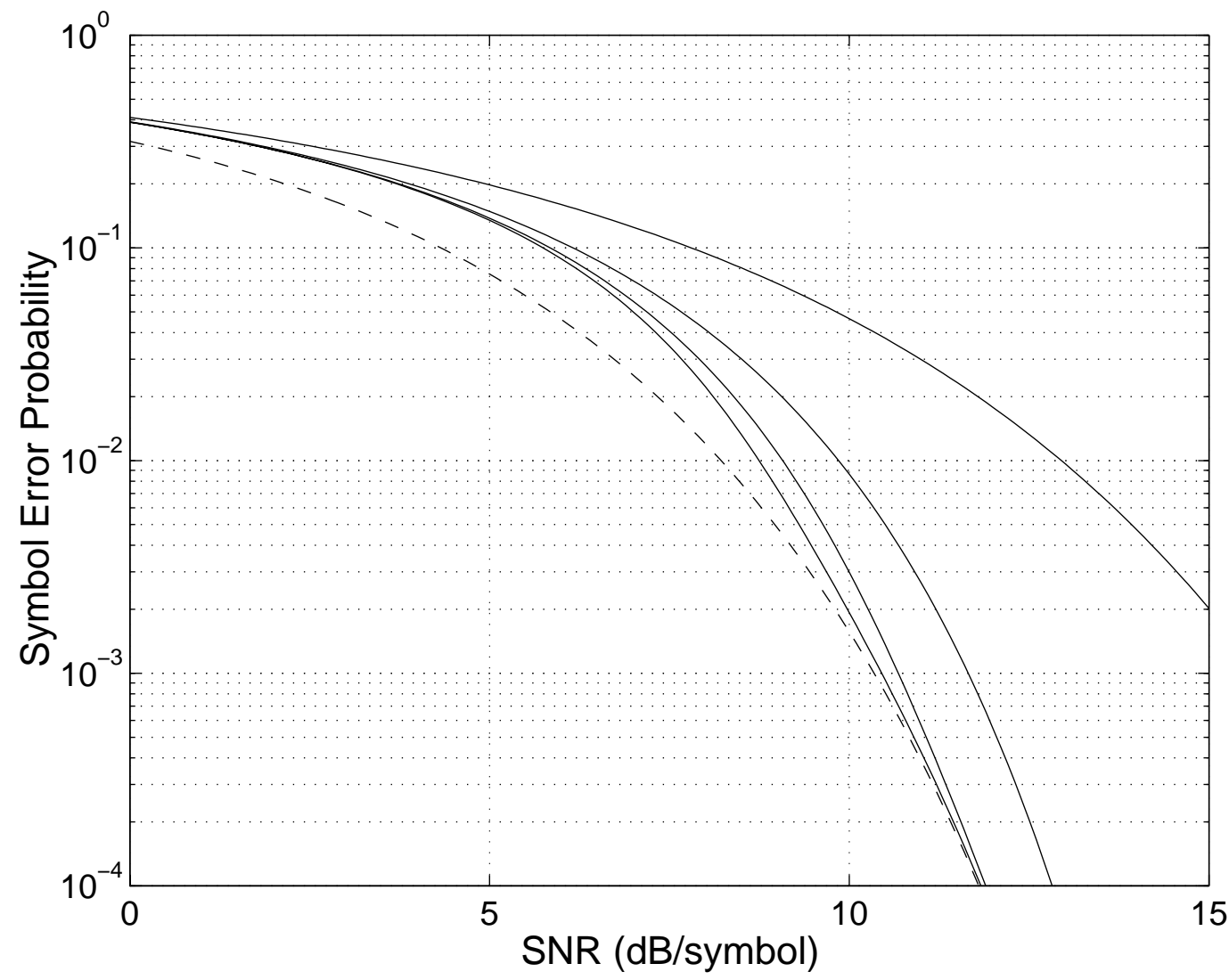

Figure 8: Iterative receiver performance as a function of SNR on the forward link. The successively lower solid curves depict the symbol error rate as a function of SNR for 1, 2, 3, 5, and $\infty$ decoding passes for QPSK transmission. The dashed curve is the symbol error rate performance on a classical additive white Gaussian noise channel with the corresponding SNR. 


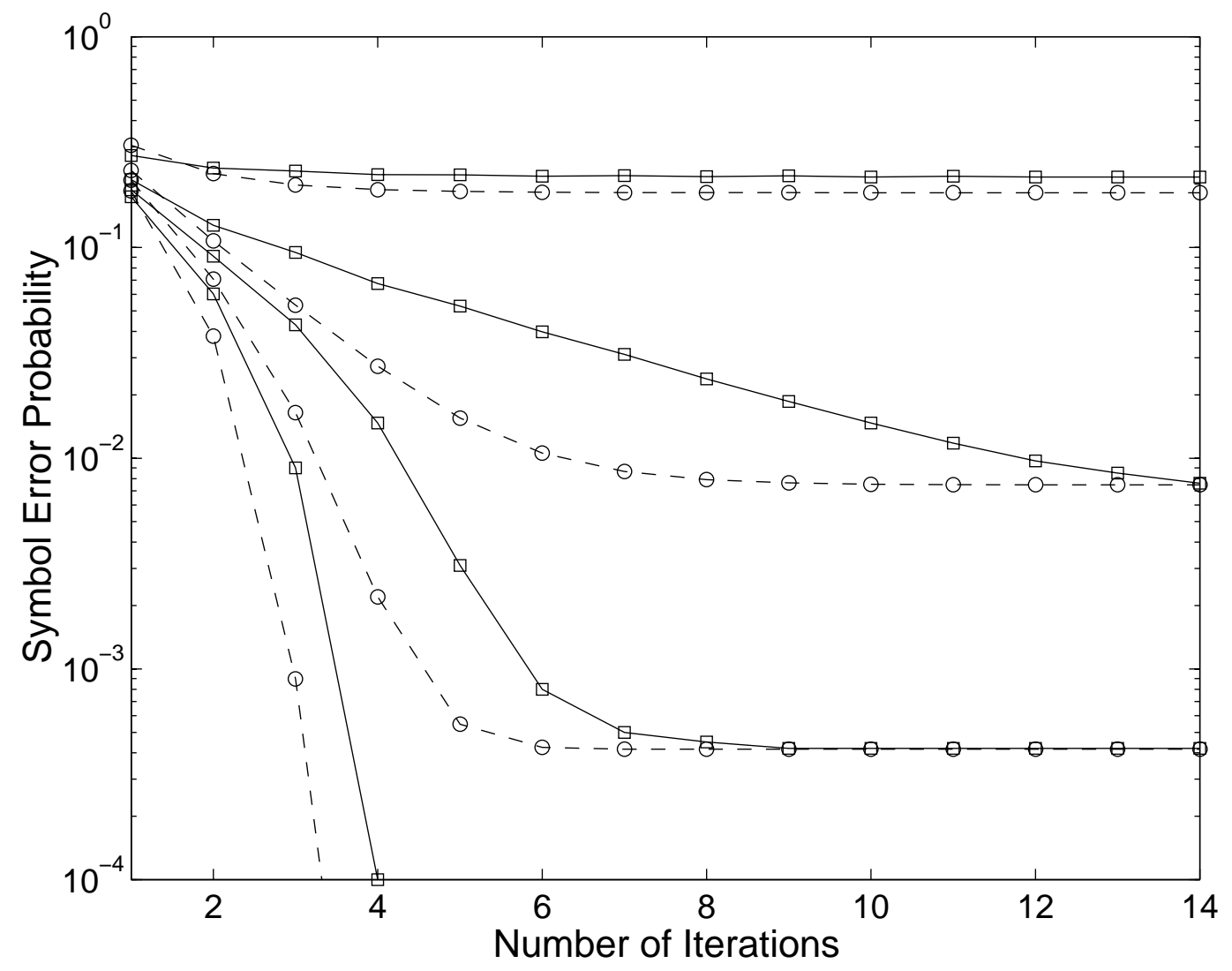

Figure 9: Iterative receiver performance on the reverse link, with $M=2$ users and power control. The successively lower curves formed from O's connected by dashed lines depict predicted QPSK symbol error rate as a function of the number of decoding passes, for SNRs of 5, 9, 11, and $14 \mathrm{~dB}$. The corresponding curves formed from $\square$ 's connected by solid lines are the experimental measurements. 


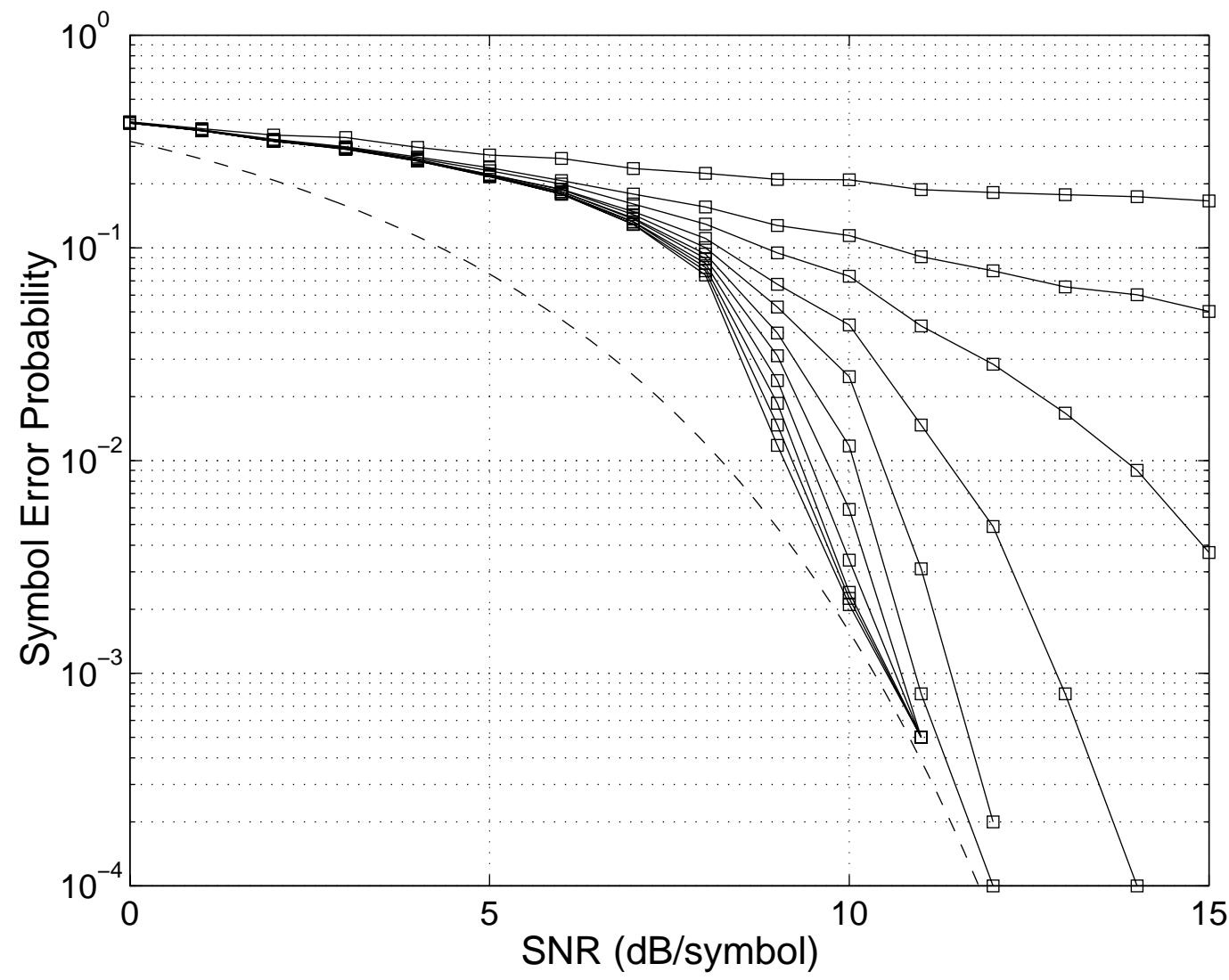

Figure 10: Iterative receiver performance as a function of SNR on the reverse link, with $M=2$ users and power control. The successively lower solid curves formed from measurements (shown as $\square$ 's) depict the QPSK symbol error rate as a function of SNR for $1,2, \ldots, 10$, and $\infty$ decoding passes. The dashed curve is the corresponding AWGN channel performance. 